\title{
Role of Healthcare Providers' Recommendation Style in HPV Vaccine Decision- Making among Haitian Parents and Female Patients
}

\author{
Dudith Pierre-Victor ${ }^{1,2}$, Dionne P. Stephens ${ }^{3}$, Rachel Clarke ${ }^{2}$, Kemesha Gabbidon ${ }^{2}$, and Purnima \\ Madhivanan ${ }^{2,4}$ \\ ${ }^{1}$ National Cancer Institute, Division of Cancer Prevention \\ ${ }^{2}$ Robert Stempel College of Public Health and Social Work, Florida International University \\ ${ }^{3}$ College of Arts and Science, Florida International University \\ ${ }^{4}$ Public Health Research Institute of India
}

\begin{abstract}
Background and Purpose: The strong influence of physician recommendation on vaccine uptake is well established in the literature. However, its influence on HPV vaccine decision-making among young Haitian women is understudied. This study investigated the role of healthcare providers' recommendation style in Haitian parents' and female patients' HPV vaccine decision-making. Methods: Thirty selfidentified Haitian women aged 17-26 years were recruited from a large university campus in the Southeastern United States (N=30). They completed in-depth face-to-face interviews regarding their HPV vaccine decision-making process. Transcripts were analyzed using thematic analysis. Results: Provider recommendation was cited as a major factor that influenced HPV vaccine uptake. Additionally, the framing of the HPV vaccine message greatly influenced vaccine decisions. Messages that framed the vaccine as a preventive measure for cervical cancer or as a preventive vaccine without an emphasis on the sexual transmission route of the virus were most effective. Messages that framed the vaccine as a preventive measure against a sexually transmitted infection did not positively influence young women to be vaccinated. Conclusions: These findings indicate that providers' recommendation style highly affects HPV vaccine decision. Thus, appropriate HPV vaccine framing is important for encouraging vaccine uptake in this ethnic group.
\end{abstract}

(C) 2017 Californian Journal of Health Promotion. All rights reserved.

Keywords: HPV vaccine; Haitian; healthcare providers; recommendation style; and decision-making

\section{Background}

Human papillomavirus (HPV) is the most common sexually transmitted infection (STI) (Centers for Disease Control and Prevention, 2016), and has been causally linked to oropharyngeal and anogenital cancers (Bosch, Lorincz, Muñoz, Meijer, \& Shah, 2002; Jones, Rowan, \& Stewart, 2005). About half of all new HPV infections occur among individuals aged 15-24 years (Centers for Disease Control and Prevention, 2016). The United States Federal Drug Administration licensed the first HPV preventive vaccine in 2006 (Centers for Disease Control and Prevention, 2010), and the vaccine confers protection against HPV strains that cause about $70 \%$ of HPV-related cancers (Dempsey, Abraham, Dalton, \& Ruffin, 2009).
The vaccine has a three-dose schedule and is recommended for boys and girls age 11-12 years. Catch-up vaccination is available until the age of 26 (Centers for Disease Control and Prevention, 2012). Additionally, the United States Healthy People 2020 has a goal of $80 \%$ coverage for HPV vaccination (U.S. Department of Health and Human Services, 2014), but currently, the overall vaccination rate is about 49\% among women aged 18-26 years (Cohen, Legg, Hutchinson, Levy, \& Bosher, 2015).

Haiti has one of the highest cervical cancer incidence and the highest associated mortality in the Western Hemisphere (International Agency for Research on Cancer, 2014). Lack of 
nationally implemented cervical cancer screening programs contribute largely to the higher incidence and mortality experienced by Haitian women (Pan American Health Organization \& World Health Organization, 2008). Haitian women residing in the United States (U.S.) continue to experience a disproportionate burden of cervical cancer despite the increased availability of screening services (Kobetz, Mendoza, \& Menard, 2010). For example, for the 2000-2004 period, the incidence of cervical cancer was 9.1/100,000 for the state of Florida while the incidence was 38.0/100,000 in Little Haiti, a Haitian community in Miami, Florida (Florida Charts, 2014). During their young adulthood, Haitian women are at high risk of acquiring HPV infections (Centers for Disease Control and Prevention, 2016). Moreover, Haitian women experience unique cultural and important socioeconomic factors that contribute to an increased risk of developing cervical cancer in the future (Pan American Health Organization \& World Health Organization, 2008;Stephens \& Thomas, 2013).

Physician recommendation for the HPV vaccine has been identified as a strong predictor of HPV vaccination among adolescents (Jeudin, Liveright, Del Carmen, \& Perkins, 2014; Mazza, Petrovic, \& Chakraborty, 2012; Perkins, Brogly, Adams, \& Freund, 2012; Reiter, Katz, \& Paskett, 2013; Stephens \& Thomas, 2013; Vadaparampil et al., 2014). Mothers are usually the parent responsible for vaccine decisionmaking. Therefore, mother-clinician interactions are among the factors that influence mothers' decision to allow their adolescent daughters to be vaccinated against HPV (Griffioen et al., 2013). Among young women, provider recommendation for the vaccine has been identified as a facilitator of HPV vaccine uptake (Chao, Velicer, Slezak, \& Jacobsen, 2010; Hopfer \& Clippard, 2011; Moore, Crosby, Young, \& Charnigo, 2010; Rosenthal et al., 2011).

Previous studies have suggested that Haitian parents highly valued the knowledge and recommendations of healthcare providers (Ryan, Hawkins, Parker, \& Hawkins, 2004; Saint-Jean
\& Crandall, 2005; Schnepel, 2007) and were more likely to have their adolescent daughters vaccinated against $\mathrm{HPV}$, if recommended by a provider (Pierre Joseph et al., 2012; Stephens \& Thomas, 2013; Wilson, Brown, Boothe, \& Harris, 2013). Additionally, healthcare providers serve as the primary source of health information for Haitian mothers (Pierre Joseph et al., 2012). Therefore, they may play a major role in HPV vaccine adoption among young Haitian women.

For any ethnic group, cultural beliefs directly or indirectly affect the acceptance of health messages and the adoption of promoted health behaviors (Hecht \& Krieger, 2006; Kreuter \& Mcclure, 2004). When a health promotion message is congruent with a group's cultural beliefs, group members are more likely to accept the message (Institute of Medicine, 2002; Kreuter \& Mcclure, 2004). Although parents across cultures view healthcare providers as a trusted source of health information and highly value their recommendation for the HPV vaccine, previous research has reported cultural differences in acceptance of the HPV vaccine based on the message framing (Lechuga, Swain, \& Weinhardt, 2011; Nan, 2012; Petrova et al., 2015). Given the high prevalence of cervical cancer among Haitian women, identifying HPV recommendation framings that are appropriate for this group can help increase HPV vaccine uptake. Hence, this study explored the role of healthcare providers' recommendation style in Haitian parents' and female patients' HPV vaccine decision-making.

\section{Methods}

We sought to understand the role of providers' recommendation style in Haitian parents' and female patients' HPV vaccine decision-making processes and to obtain a rich description of their HPV decision-making processes in a cultural context. Qualitative approaches allow for more in-depth and nuanced description of participants' and parents' decision-making processes as they relate to the messages used by their health care providers. 
Pierre-Victor, D., Stephens, D., Clarke, R., Gabbidon, K., Madhivanan, P./ Californian Journal of Health Promotion 2017, Volume

\section{Participants}

Recruitment for this study took place at a large minority-serving university in Southeastern United States. Thirty participants were recruited via an online psychology research pool known as 'Sona'. Through Sona, students enrolled in psychology courses were screened for eligibility prior to scheduling their interviews; criteria included self-identifying as Haitian, female, and being between 17 and 26 years of age. We wanted to interview Haitian females who were still eligible for the vaccine, as indicated by the Advisory Committee on Immunization Practices guidelines (Centers for Disease Control and Prevention, 2013). We sought to interview vaccinated and unvaccinated Haitian females, whether the decision was made by their parents (with or without their input) or by themselves as young adults. Therefore, potential participants were not screened based on HPV vaccination status and timing of vaccine uptake. Females who were no longer eligible for the vaccine (older than 26 years) and those who did not selfidentify as Haitian or Haitian American were restricted from signing up for the study in Sona.

\section{Procedures}

The principal investigator conducted the individual interviews at a time selected by the participants. Study participants underwent a consent process which included reading the letter of consent approved by the university's Internal Review Board and asking questions to the principal investigator. Participants then completed a demographic questionnaire that collected the participant's age, self-identity, current class standing, country of birth, number of years in the U.S., and parents' country of birth. For the few participants who were younger than 18, their parent's consent was obtained prior to the interview.

A series of semi-structured yet flexible questions were developed based on qualitative HPV vaccine research conducted among college students (Hopfer \& Clippard, 2011), Haitian and African American parents (Pierre Joseph et al., 2012), Haitian parents (Stephens \& Thomas, 2013), and ethnically diverse Black women (Wilson et al., 2013). Similar to previous research in the field (Stephens \& Thomas, 2013; Wilson et al., 2013), interviews began with questions to establish HPV vaccination knowledge and beliefs (Appendix I). The interview captured participants' HPV infection and vaccine knowledge, source of information, HPV vaccine perceptions and attitudes, vaccination status, HPV vaccine facilitators, and barriers to HPV vaccination. More time was dedicated toward HPV vaccine facilitators (for those who have been vaccinated), barriers to HPV vaccination (for those who were not vaccinated), and decision-making process compared to other constructs. Interview questions included: Have you ever heard of Human Papillomavirus or HPV? Where/how did you hear about it? What do you know about the vaccine? Where/how did you get this information? Who should get the HPV vaccine? Have you initiated the HPV vaccine series? What/who motivated you to get the vaccine? And what/who do you think influenced you to not get the vaccine?

Interviews were conducted in a private conference room; with each session audio-taped, with the shortest interview lasting 15 and the longest lasting 65 minutes. Interview length varied by participants' level of HPV awareness, experience with HPV vaccine messages, and decision-making process. The interviews were conducted in English, between June 2014 and March 2015.

\section{Data Analyses}

A thematic analysis was undertaken. Data collection and analysis proceeded simultaneously (Corbin \& Strauss, 2014). The interviews were transcribed, and then verified by one study investigator to ensure completeness, accuracy of the discussion content, and a high quality of transcription. A preliminary coding framework related to HPV vaccine knowledge and attitudes was constructed by three study authors after an in-depth reading of the transcripts. Themes identified in this first stage were not specified a priori but rather were derived from the data. Meaningful units were selected by the research team, these units were coded and used to develop the preliminary coding framework. Codes were then used to 
develop overarching themes. Additional codes were developed inductively and new themes emerge through the analysis process. Themes and subthemes were then examined across the whole dataset, and in relation to each specific interview. The research team used a process of negotiated agreement to determine inter-rater agreement which included meeting to discuss and further refine each set of themes, resolved differences, and reached consensus on a coding scheme. Discrepancies were resolved by first revisiting and reviewing the data, and then through group discussion. Key themes were summarized, reviewed, and agreed upon by the research team.

\section{Results}

A total of 30 young women with a median age of 21 participated in the study. Although ten of the participants were born abroad, only four had been living in the United States for fewer than ten years. Eighteen had not received the vaccine, eight had begun the HPV vaccine series, and four had completed the series (Table 1). The results presented here are organized around three major themes identified in the analysis of the role of healthcare providers' recommendation style in Haitian parents' and female patients' HPV vaccine decision-making: 1) provider recommendation, 2) HPV vaccine message framing, and 3) vaccine decision. The message framing and the decision following the vaccine recommendation are presented jointly. For the purposes of this study, a HPV vaccine recommendation is defined as: 1) any discussion in which a healthcare provider informs the patient and/or parents about the vaccine and 2) offers the vaccine, positively endorses it, or directly recommends it. Following each quote, the participant's status for parental consent at the time of the recommendation is provided.

\section{Provider Recommendation}

Sixteen of the 30 participants stated that they have seen a healthcare provider and have had a discussion regarding the vaccine. Of the remaining 14 participants, 12 stated that they never received a recommendation from a provider, and two stated that they could not recall having such a discussion with any of their healthcare providers. The results presented below are from the 16 participants who have received a provider recommendation for the vaccine.

Table 1

\begin{tabular}{lll}
$\begin{array}{l}\text { Demographic Characteristics } \\
\text { Participants }\end{array}$ & of & Study \\
\hline Characteristics & $\mathrm{n}$ & $\%$ \\
\hline Self-Identity & 10 & 33 \\
Haitian & 20 & 67 \\
Haitian-American & & \\
Class Standing & 15 & 50 \\
Seniors /Graduate & 5 & 17 \\
Sophomore/Junior & 10 & 33 \\
Freshman & & \\
Country of Birth & 23 & 70 \\
USA & 7 & 30 \\
Haiti & & \\
HPV Vaccination Status & 18 & 60 \\
Unvaccinated & 8 & 27 \\
Initiated series & 4 & 13 \\
Completed series & &
\end{tabular}

Twelve of the 16 participants who have received at least one provider recommendation for the vaccine were vaccinated. When asked about who or what motivated them to get the vaccine $(n=12)$, one stated she received it because her school required it, and the other 11 cited healthcare provider recommendation among the factors that highly motivated them to take the vaccine. Most participants who had HPV vaccine discussion had it with a physician $(\mathrm{n}=13)$, and a few young women had such discussion with a nurse $(n=3)$. Two participants stated that they received a recommendation for the vaccine on two separate occasions.

The doctor said it [the vaccine] was recommended. (Pcpt 3, minor)

When I went to get a check-up at my doctor's office. He asked my mom if she wanted me to have the human papillomavirus shot. (Pcpt 10, minor)

The doctor told me I had to take the vaccine. (Pcpt 11, minor) 
Pierre-Victor, D., Stephens, D., Clarke, R., Gabbidon, K., Madhivanan, P./ Californian Journal of Health Promotion 2017, Volume 15, Issue 3, Pages 68-80.

He [the doctor] told me that he had no daughters... But if he had daughters, he would highly recommend it [the vaccine]. (Pcpt 13, minor)

I went for a routine check-up at the doctor's office, and she asked my parents if I wanted to get an HPV shot. (Pcpt 20, minor)

I was at the doctor's office and she recommended that I took it [the vaccine]. (Pcpt 25, minor)

I went for school vaccine, and she [the nurse at the Student Health Clinic] offered it [the vaccine] to me. (Pcpt 15, young adult)

The 16 participants who had HPV vaccine discussion were asked who was present at the time of the discussion. Twelve had the HPV vaccine discussion as minors and six had the vaccine discussion as young adults while attending college. All the participants who had the conversation as minors $(n=12)$ were with a parent at the time of the recommendation. Nine of them were with their mother. Of the remaining three, one was with her father, one with her stepmother, and one with both parents. Of those six participants who had the vaccine discussion as young adults while attending college, three received the recommendation from a nurse. Additionally, one of those six participants was a young adult accompanied by her mother at the time of the recommendation (Pcpt 5).

\section{HPV Message Framing and Vaccine Decision}

Participants who received a physician recommendation for the vaccine were asked to comment on the HPV vaccine message they received, the decision following the recommendation, and specific details that led to their vaccine decision. While more than half of the young women had been recommended the vaccine by a healthcare provider $(n=16)$, the framing of the vaccine recommendation was not uniform, irrespective of the type of provider (physician vs. nurse). Young women, adolescent females, and their parents responded differently to the recommendation depending on the vaccine message framing. Less than half of the healthcare providers framed the HPV vaccine as a cervical cancer prevention vaccine $(n=6)$. When the message was framed as a cervical cancer prevention vaccine, parents had the tendency to respond favorably to the provider recommendation.

They say that I should take it [the vaccine], that it would help prevent cervical cancer. She [my mother] said "okay", and she asked me if I was fine with it. We had cancer in the family before, and we lost a family member to cancer... I think anything that deals with cancer, we take precautions with that. It is hard to lose someone [to cancer], and then to lose a child would even be harder. (Pcpt 21, minor)

The doctor told my dad and me what the vaccine was and what it was meant to prevent [cervical cancer], as well as, the vaccine was recommended for girls my age. I think just because the doctor said it. I don't think my dad knows what HPV is. Once he [my father] heard cancer, he decided on the vaccine to take precautions. (Pcpt 3, minor)

He [doctor] just said it [the vaccine] was important to try to prevent cervical cancer. I think he could have explained more because he just said it prevents cervical cancer and that was it. He didn't really go into details about it. She [my mom] said that if it's a health benefit then I should take it. I wanted to take it, and it was my decision. (Pcpt 5, young adult)

More than half of the 16 participants received a HPV vaccine recommendation that was not framed as a cervical cancer prevention measure but as a recommended vaccine for HPV infection prevention $(n=9)$. These messages included information regarding the sexual transmission route of the virus. Parents responded positively when the sexual transmission route was not emphasized. Two participants pointed out that the physician explained to their mothers that the virus was not transmitted solely through sexual intercourse. They stated that this information probably influenced their mothers' decision to get them vaccinated. For the remaining seven participants, several of them reported that their parents raised side effect concerns due to the newness of the vaccine compared to other adolescent vaccines or declined it based on the sexual activity status of the adolescent.

We were both in the office, and she [the doctor] asked me if I wanted to take it [HPV vaccine]. 
Pierre-Victor, D., Stephens, D., Clarke, R., Gabbidon, K., Madhivanan, P./ Californian Journal of Health Promotion 2017, Volume 15, Issue 3, Pages 68-80.

And she [the doctor] was explaining the benefits of the vaccine and the symptoms of the infection and the facts. And my mom was like "She does not need it. I do not see a need for her to take it." So I did not take it at that time. My mom knows I am not sexually active. That is why she did not see a need for me to get it. I may take it, but I am not sexually active right now. So, I don't see the need for it. (Pcpt 29, minor)

He [the doctor] just told us about HPV and that the vaccine was fairly new. The vaccine was offered to me when I was 15 turning 16 or just turned 16. And she [my mom] was like "I am not sure yet. Let's see how everybody else reacts to it. It is so new. You don't know what happens after people take it." .... She was like "This is my decision...As far as I know, you are not sexually active, so I don't think you need it." (Pcpt 17, minor)

He [doctor] was talking about it. He was like "There is a shot for it, and it is better if you take it than if you do not take it. You do not have to be sexually active to get the virus. It is better to take the vaccine if you are not engaged in sexual activity than if you are. If you engaged in sexual activity, the risk of infection is higher." So probably that is why my mom was like "Let me [have the doctor] give it to the girls before they get sexually active." ...We were talking about it, but we did not make the decision right then and there. I think we made the decision after a few days to about a week. My mom was hesitant at first because you know everyone has different reaction to [the vaccine]. And she was like "It is up to you if you want to do it. It does not seem bad". So, I thought about it and I was like 'yeah'. And [we] went back to get it. (Pcpt 14, minor)

When I went to get a check-up at my doctor's office they asked my mom if she wanted me to have the human papillomavirus shot. It was the same pediatrician's office since I was a baby. I think the doctor made her aware that it can be transmitted sexually and in other ways too. she decided it would be a good idea. She [my mom] said "Sure, why not?" But since she's adamant about me not having sex in the near future, the other ways probably concerned her more so that's why [she agreed]. (Pcpt 10, minor)
A few participants mentioned that their healthcare provider recommended that they be vaccinated against HPV if they were sexually active $(n=3)$. This message framing was not received favorably among parents and adolescent girls. Participants to whom the vaccine was framed as such stated that they did not receive the vaccine following the recommendation. One participant stated that the healthcare provider advised her to take the vaccine if she was sexually active while her mother was in the examination room. The provider handed or pamphlet about HPV to the adolescent. That participant specifically said that she was discontented with the healthcare provider discussing her sexual activity status in her mother's presence. A young woman who received a vaccine message framed in this manner declined the vaccine due to side effects concerns based on a previous experience with another vaccine and the fact that several trips were required to complete the series.

The doctor said they had it [HPV vaccine] and if I am sexually active she recommends that I take it. She asked if I am sexually active. She [my mother] looked at me to see what I was going to say. I said I was not going to take the vaccine because I am not sexually active, and I do not plan to be sexually active anytime soon. And it was kind of awkward. They gave me a pamphlet and I just went home...She was having that conversation with me in front of my mom. It was weird. (Pcpt 28, minor)

I went to a clinic to do some basic check-up for a job. A nurse asked me if I am interested in getting the HPV vaccine. She asked me if I was sexually active and I told her 'I wasn't'. She asked me "Have [you] been in the past?" I told her 'yes'. She [a nurse] said I should get it [the vaccine]. And she was like 'Why not get it now because it is better to get it now because if you go and do have sex.... I thought 'you know what? This is something I need to think about'. Because at the time I was not sexually active, and I thought 'Let me look into it, and then I would make my decision'. She was trying to convince me to get it, but I remembered the side effects of the flu shot. ... She explained the potential side effects, and they were a little scary...I knew I had to go back more than once, and I did not want to go back more than once. I 
Pierre-Victor, D., Stephens, D., Clarke, R., Gabbidon, K., Madhivanan, P./ Californian Journal of Health Promotion 2017, Volume

15, Issue 3, Pages 68-80.

did not like that fact and had to think about it because everything has advantages and disadvantages. (Pcpt 8, young adult)

\section{Discussion}

The goal of this study was to investigate the role of healthcare providers' recommendation style in Haitian parents' and female patients' HPV vaccine decision-making. Study participants commented on the central role of provider recommendation in their parents' decision to get them vaccinated as minors and in their own decision to be vaccinated as young adults. This finding is anticipated given that healthcare provider recommendation has been consistently associated with HPV vaccine uptake crossculturally (Jeudin et al., 2014; Mazza et al., 2012; Perkins et al., 2012; Reiter et al., 2013; Vadaparampil et al., 2014).

In this study, mothers were the primary parental figure who most often accompanied their daughters during their healthcare providers' visit. Several studies have attested to the important role of clinician-mother interaction in HPV vaccine decision-making (Dempsey et al., 2009; Gerend, Weibley, \& Bland, 2009; Griffioen et al., 2013). Following the motherclinician conversation about the vaccine, generally, the mothers shared their endorsement of the vaccine with their daughters and told them to take it or they expressed the opposition or concern directly to the provider.

Study findings revealed that recommendation style varied among healthcare providers, and is of crucial importance in HPV vaccine decisionmaking. Some healthcare providers framed the vaccine as a cervical cancer prevention vaccine. This framing was well-received by Haitian parents, and they often perceived the vaccine as beneficial. These results concur with those of a previous study that reported significantly higher intentions among mothers to get their daughter vaccinated following a HPV vaccine message focusing on cervical cancer protection (Lechuga et al., 2011). In our study, a participant receives a recommendation framed in this manner as a young adultaccompanied by her mother. Her mother endorsed the vaccine following the message, and she accepted it. However, being a young adult, she viewed the decision as her own. Higher vaccine acceptance has been reported among young women when HPV message framing focused on cervical cancer prevention (Leader, Weiner, Kelly, Hornik, \& Cappella, 2009). Additionally, another study showed that more young men intended to get the vaccine when it was framed as a cancer prevention vaccine as compared to a genital warts preventive measure (McRee, Reiter, Chantala, \& Brewer, 2010). One participant had lost a family member to cancer, and her mother responded positively to this framing. Women who experience cervical disease or cervical cancer are more highly motivated to have their daughters vaccinated against HPV (Hamlish, Clarke, \& Alexander, 2012). Among participants to whom the vaccine was framed in this manner, a young adult mentioned the provider could have provided more information. To ensure higher vaccine acceptance, providers should take the time to provide information regarding the vaccine. Along with findings from the studies cited above, these results indicate that the cancer prevention framing increases HPV vaccine acceptance and uptake.

A group of providers framed the vaccine as a preventive measure against HPV infection and informed parents and participants that the virus was transmitted sexually. Two participants reported that their physicians emphasized that HPV is not transmitted only through sexual intercourse. Parents did not oppose the vaccine when messages were framed in this manner. Upon hearing that the virus was not transmitted solely through sexual intercourse, the mothers consented for the vaccine. Although HPV has been detected in vaginal swabs from virginal women (Bumbuliene, Alisauskas, \& Sepetiene, 2011; Eleutério et al., 2013; Winer et al., 2003), participants did not comment on the other potential route of transmission and were not probed further to assess whether the providers explained the other potential routes of transmission. Nevertheless, informing parents that the virus was not transmitted solely through sexual intercourse convinced them to get their daughters vaccinated. Other participants to whom the vaccine was framed as a 
recommended vaccine for a sexually transmitted infection reported that their parents responded by raising safety concerns because the HPV vaccine has not been licensed for as long as the other adolescent vaccines or by informing the providers that their daughters did not need the vaccine. Previous studies have reported side effect concerns among parents (Laz, Rahman, \& Berenson, 2012; Sanders Thompson, Arnold, \& Notaro, 2012; Wilson et al., 2013). In this study, parents often raised side effects questions when the benefits of the vaccine were not emphasized.

A few providers asked participants about their sexual activity status before recommending the vaccine. When the vaccine was framed as a preventative measure for sexually active individuals, the vaccine recommendation was not received favorably by the parents or the adolescents. The vaccine was often declined when it was framed in this manner. Existing literature has reported that young Haitian women are expected to remain virgins until marriage (Archibald, 2007; Stephens \& Thomas, 2013). Parents may experience difficulties in encouraging preventive practices if such practices are strongly linked to sexual behaviors when there are cultural expectations of chastity for girls. In this context, discussing sexual activity status may negatively affect patientclinician, mother-clinician, and parent-daughter relationships. This message framing is likely to be met with resistance, as it is not congruent with Haitian cultural values. However, in the example given of Participant 8, to whom the vaccine was framed as such, she was not with a parent at the time of the recommendation, and she did not explicitly report feeling offended. Nevertheless, the framing of the discussion was not effective in motivating her to receive the vaccine.

This study focused on the role of healthcare providers' recommendation style in Haitian parents' and female patients' HPV vaccine decision-making in a region where there is a large Haitian population. As students at a large minority serving state university, they have access to a university health center that includes a clinic specifically serving women's sexual health needs. They can seek out sexual health resources and information outside of their familial and community spaces. Thus, these women represented a population that is distinctly different from community samples that may face greater barriers to seeking health services and information or that may have lower English proficiency. Additionally, the exact timing of participant's encounter with their provider was not captured. We simply captured whether the participants' encounter with the healthcare provider occurred while they were a minor or a young adult. Thus, recall bias may influence our results. Moreover, participants often alluded to the gender of the provider and sometimes provided the specialty, but provider demographics were not collected in the study. Future research should examine the role of the provider's gender and ethnicity, as such research might provide additional insights into the patient-provider and mother-provider interactions. A few participants stated that the provider informed their parents that HPV could be transmitted through means other than sexual intercourse. However, we did not ask participants whether the providers stated the other potential means of transmission, and such information would provide rich details regarding the message framing. Furthermore, some participants mentioned their sexual activity status at the time of the recommendation, but their sexual activity status at the time of the study and at the time of the recommendation was not collected due to the sensitivity of this issue in the Haitian culture. Therefore, participants could not be probed specifically about the influence of their sexual activity status on their vaccine decision-making. Despite these limitations, this study provides an important first step towards adding to what is currently a very small body of research addressing the role of healthcare providers' recommendation style in HPV vaccine decision-making processes among Haitian parents and female patients.

\section{Implications for Practice}

Across cultures and ethnic groups, recommendation from a healthcare provider is a highly influential factor in HPV vaccine uptake. This study suggests that HPV recommendation framing had a great influence on mothers' HPV vaccine decision for their daughter, and young 
Haitian women's decision to be vaccinated. Thus, it is important for healthcare providers to understand that the message framing is as important as the recommendation, and that a message framing that is not appropriate can result in missed opportunities for vaccination. Presenting HPV vaccine as a HPV-related cancer or cervical cancer preventive vaccine may motivate parents and young women to consider the long-term benefits of the vaccine. Should providers prefer to present the vaccine to Haitian parents as a preventive measure against HPV infection, they should explain the link between the virus cancer and should avoid emphasizing the sexual transmission route. Considering the cultural taboo of discussing sex with Haitian teens primarily in the presence of parents, it may be more useful and comfortable for health providers to discuss teens' sexual activity outside of parents' presence. In the presence of a Haitian parent, framing HPV vaccine as a STI preventive vaccine for the sexually active will severely reduce vaccine acceptability for the adolescent. If accompanied by a parent and the vaccine is framed as a STI preventive measure for the sexually active, a young Haitian woman would not feel comfortable accepting the vaccine should she be sexually active, as young Haitian women are expected to remain virgins until marriage (Archibald, 2007; Stephens \& Thomas, 2013). Additionally, providers should be reminded that teens' level of sexual activity is not relevant to vaccine recommendation. Providers should refrain from asking patients about their sexual activity status in the context of HPV vaccine recommendation. Instead, providers should emphasize that the vaccine is best taken prior to initiating sexual activity. The vaccine is recommended for adolescents and young adults ideally prior to their initiation of sexual activity, and providers should recommend it that way. Recommending the vaccine specifically to sexually active individuals may lead to the conclusion that the vaccine is for the sexually promiscuous and would likely result in a missed opportunity for vaccination. Moreover, future studies should investigate parents' and young adults' response to HPV vaccine recommendation from nurses and physician assistants, as a few participants had received a recommendation for the vaccine from nurses in settings other than doctor's office.

\section{Conclusion}

Cross-culturally, healthcare provider recommendation plays a crucial role in influencing HPV vaccine uptake. As evidenced by these findings, provider recommendation style influences parents' and female patients' decision-making following the provider recommendation. Consequently, when framing the HPV vaccine messages, healthcare providers should consider the patient's culture as culturally inappropriate vaccine messages can reduce vaccine acceptability. Additionally, providers should invite parents as well as the adolescents to ask any questions and raise any concerns they may have, and that would be an opportunity to provide additional information, refute vaccine misconceptions, and address side effect concerns.

\section{Acknowledgements}

The authors are thankful to Newsoul Lapaix, Dr. Linda Bliss, and colleagues in the Social and Health Disparities Research Lab for their guidance in this research endeavor. We thank all participants for their time and willingness to take part in the study.

Funding Source

This research was supported by the Florida Education Fund through the McKnight Doctoral Fellowship (2014-2015).

\section{Disclaimer}

The findings and conclusions in this manuscript are those of the authors and do not represent the official position of the National Cancer Institute. 
Pierre-Victor, D., Stephens, D., Clarke, R., Gabbidon, K., Madhivanan, P./ Californian Journal of Health Promotion 2017, Volume 15, Issue 3, Pages 68-80.

\section{References}

Archibald, C. (2007). Knowledge and attitudes toward HIV/AIDS and risky sexual behaviors among Caribbean African American female adolescents. Journal of the Association of Nurses in AIDS Care, 18(4), 64-72. https://doi.org/10.1016/j.jana.2007.05.004

Bosch, F. X., Lorincz, A., Muñoz, N., Meijer, C. J. L. M., \& Shah, K. V. (2002). The causal relation between human papillomavirus and cervical cancer. Journal of Clinical Pathology, 55, 244-265.

Bumbuliene, Z., Alisauskas, J., \& Sepetiene, A. (2011). High-risk hpv infection in sexually active and non active young women. Central European Journal of Medicine, 6(4), 456-462. https://doi.org/10.2478/s11536-011-0042-0

Centers for Disease Control and Prevention. (2010). FDA licensure of quadrivalent human papillomavirus vaccine (HPV4, Gardasil) for use in males and guidance from the Advisory Committee on Immunization Practices (ACIP). MMWR, 56(20), 630-632.

Centers for Disease Control and Prevention. (2012). Advisory Committee on Immunization Practices: human papillomavirus vaccine resolution. Retrieved March 13, 2015, from http://www.cdc.gov/vaccines/programs/vfc/providers/resolutions.html

Centers for Disease Control and Prevention. (2013). The Advisory Committee on Immunization Practices (ACIP). Retrieved from http://www.cdc.gov/vaccines/acip/about.html

Centers for Disease Control and Prevention. (2016). Genital HPV Infection- CDC Fact Sheet. Retrieved September 16, 2016, from https://www.cdc.gov/std/hpv/stdfact-hpv.htm

Chao, C., Velicer, C., Slezak, J. M., \& Jacobsen, S. J. (2010). Correlates for human papillomavirus vaccination of adolescent girls and young women in a managed care organization. American Journal of Epidemiology, 171(3), 357-367. https://doi.org/10.1093/aje/kwp365

Cohen, T., Legg, J. S., Hutchinson, J., Levy, J., \& Bosher, W. (2015). Factors influencing HPV vaccine use among racially diverse female college students. Journal of Health Disparities Research and Practice, 8(2), 75-88.

Corbin, J., \& Strauss, A. (2014). Basics of qualitative research: Techniques and procedures for developing grounded theory. Thousand Oaks, CA: Sage.

Dempsey, A. F., Abraham, L. M., Dalton, V., \& Ruffin, M. (2009). Understanding the reasons why mothers do or do not have their adolescent daughters vaccinated against human papillomavirus. Annals of Epidemiology, 19(8), 531-538. https://doi.org/10.1016/j.annepidem.2009.03.011.Understanding

Eleutério, M. R. N., de Oliveira, M. P., Jacyntho, C. M. A., Rodrigues, J. F., Cavalcante, I. D. M., \& Eleutério, J. J. (2013). Prevalence of HPV in adolescents virgins and sexually active at a university hospital in the city of Rio de Janeiro, Brazil. ISRN Infectious Diseases. Retrieved from http://dx.doi.org/10.5402/2013/387961\%0AClinical

Florida Charts. (2014). Cervical Cancer Incidence. Retrieved September 1, 2014, from http://www.floridacharts.com/charts/OtherIndicators/NonVitalIndDataViewer.aspx?cid=0449

Gerend, M. A., Weibley, E., \& Bland, H. (2009). Parental response to human papillomavirus vaccine availability: Uptake and intentions. Journal of Adolescent Health, 45(5), 528-531. https://doi.org/10.1016/j.jadohealth.2009.02.006

Griffioen, A. M., Glynn, S., Mullins, T. K., Zimet, G. D., Rosenthal, S. L., Fortenberry, J. D., \& Khan, J. A. (2013). Perspectives on decision making about human papillomavirus vaccination among 11to 12-year-old girls and their mothers. Clinical Pediatrics, 51(6), 560-568. https://doi.org/10.1177/0009922812443732.Perspectives

Hamlish, T., Clarke, L., \& Alexander, K. A. (2012). Barriers to HPV immunization for African American adolescent females. Vaccine, 30(45), 6472-6476. https://doi.org/10.1016/j.vaccine.2012.07.085

Hecht, M. L., \& Krieger, J. L. R. (2006). The principle of cultural grounding in school-based substance abuse prevention. Journal of Language and Social Psychology, 25(3), 301-319. 
Pierre-Victor, D., Stephens, D., Clarke, R., Gabbidon, K., Madhivanan, P./ Californian Journal of Health Promotion 2017, Volume 15, Issue 3, Pages 68-80.

Hopfer, S., \& Clippard, J. R. (2011). College women's HPV vaccine decision narratives. Qualitative Health Research, 21(2), 262-77. https://doi.org/10.1177/1049732310383868

Institute of Medicine. (2002). Speaking of health: Assessing health communication stratefies for diverse populations. Washington, D.C.

International Agency for Research on Cancer. (2014). GLOBOCAN 2012: Estimated cancer incidence, mortality and prevalence worldwide in 2012. IARC CancerBase. Lyon, France. Retrieved from http://globocan.iarc.fr/Pages/fact_sheets_cancer.aspx

Jeudin, P., Liveright, E., Del Carmen, M. G., \& Perkins, R. B. (2014). Race, ethnicity, and income factors impacting human papillomavirus vaccination rates. Clinical Therapeutics, 36(1), 24-37. https://doi.org/10.1016/j.clinthera.2013.11.001

Jones, R. W., Rowan, D. M., \& Stewart, A. W. (2005). Vulvar intraepithelial neoplasia: Aspects of the natural history and outcome in 405 women. Obstetrics and Gynecology, 106(6), 1319-1326.

Kobetz, E., Mendoza, A. D., \& Menard, J. (2010). One Size Does Not Fit All : Differences in HPV Knowledge between Haitian and African American Women One Size Does Not Fit All : Differences in HPV Knowledge between Haitian and African. Cancer Epidemiology, Biomarkers \& Prevention, 19(2), 366-370. https://doi.org/10.1158/1055-9965.EPI-09-1180

Kreuter, M. W., \& Mcclure, S. M. (2004). The role of culture in health communication. Annual Reviews of Public Health, 25, 439-455. https://doi.org/10.1146/annurev.publhealth.25.101802.123000

Laz, T. H., Rahman, M., \& Berenson, A. B. (2012). An update on human papillomavirus vaccine uptake among 11-17 year old girls in the United States: National Health Interview Survey, 2010. Vaccine, 30, 3534-40. https://doi.org/10.1016/j.vaccine.2012.03.067

Leader, A. E., Weiner, J. L., Kelly, B. J., Hornik, R. C., \& Cappella, J. N. (2009). Effects of information framing on human papillomavirus vaccination. Journal of Women's Health, 18(2), 225-233. https://doi.org/10.1089/jwh.2007.0711

Lechuga, J., Swain, G. R., \& Weinhardt, L. S. (2011). Impact of framing on intentions to vaccinate daughters against HPV: a cross-cultural perspective. Annals of Behavioral Medicine, 42(2), 2216. https://doi.org/10.1007/s12160-011-9273-1

Mazza, D., Petrovic, K., \& Chakraborty, S. (2012). HPV vaccination of adult women: an audit of Australian general practitioners. The Australian \& New Zealand Journal of Obstetrics \& Gynaecology, 52(6), 528-33. https://doi.org/10.1111/ajo.12002

McRee, A.-L., Reiter, P. L., Chantala, K., \& Brewer, N. T. (2010). Does framing human papillomavirus vaccine as preventing cancer in men increase vaccine acceptability? Cancer Epidemiology Biomarkers and Prevention, 19(8), 1937-1944. https://doi.org/10.1158/1055-9965.EPI-09-1287

Moore, G. R., Crosby, R., Young, A., \& Charnigo, R. (2010). Low rates of free human papillomavirus vaccine uptake among young women. Sexual Health, 7(3), 287-290. Retrieved from http://ovidsp.ovid.com/ovidweb.cgi?T=JS\&PAGE=reference $\& D=e m e d 9 \& N E W S=N \& A N=2010$ 464590

Nan, X. (2012). Communicating to young adults about HPV vaccination: consideration of message framing, motivation, and gender. Health Communication, 27(1), 10-18. https://doi.org/10.1080/10410236.2011.567447

Pan American Health Organization \& World Health Organization. (2008). Regional strategy and plan of action for cervical cancer prevention and control. Washington, D.C.

Perkins, R. B., Brogly, S. B., Adams, W. G., \& Freund, K. M. (2012). Correlates of human papillomavirus vaccination rates in low-income, minority adolescents: a multicenter study. Journal of Women's Health, 21(8), 813-20. https://doi.org/10.1089/jwh.2011.3364

Petrova, D., Brunton, C. G., Jaeger, M., Lenneis, A., Muñoz, R., Garcia-retamero, R., \& Todorova, I. (2015). The views of young women on HPV vaccine communication in four European countries. Current HIV Research, 13, 347-358.

Pierre Joseph, N., Clark, J. A., Bauchner, H., Walsh, J. P., Mercilus, G., Figaro, J., ... Perkins, R. B. (2012). Knowledge, attitudes, and beliefs regarding HPV vaccination: Ethnic and cultural 
differences between African-American and Haitian immigrant women. Women's Health Issues, 22(6), e571-e579. https://doi.org/10.1016/j.whi.2012.09.003

Reiter, P. L., Katz, M. L., \& Paskett, E. D. (2013). Correlates of HPV vaccination among adolescent females from Appalachia and reasons why their parents do not intend to vaccinate. Vaccine, 31(31), 3121-5. https://doi.org/10.1016/j.vaccine.2013.04.068

Rosenthal, S. L., Weiss, T. W., Zimet, G. D., Ma, L., Good, M. B., \& Vichnin, M. D. (2011). Predictors of HPV vaccine uptake among women aged 19-26: Importance of a physician's recommendation. Vaccine, 29(5), 890-895. https://doi.org/10.1016/j.vaccine.2009.12.063

Ryan, E. R., Hawkins, W. E., Parker, M., \& Hawkins, M. J. (2004). Perceptions of access to U. S. health care of Haitian immigrants in South Florida. Florida Public Health Review, 1, 30-35.

Saint-Jean, G., \& Crandall, L. A. (2005). Utilization of preventive care by Haitian immigrants in Miami, Florida. Journal of Immigrant Health, 7(4), 283-292. https://doi.org/10.1007/s10903-005-5125-z

Sanders Thompson, V. L., Arnold, L. D., \& Notaro, S. R. (2012). African American parents' HPV vaccination intent and concerns. J Health Care Poor Underserved, 23(1), 290-301. Retrieved from http://www.ncbi.nlm.nih.gov/pmc/articles/PMC3601894/?tool=pubmed

Schnepel, E. M. (2007). Stigma, status, and hidden health problems : Starting a public health dialogue among Haitians in New York City. Practicing Anthropology, 29(2).

Stephens, D. P., \& Thomas, T. L. (2013). Cultural values influencing immigrant Haitian mothers' attitude toward human papillomavirus vaccination for daughters. Journal of Black Psychology, 39(2), 156-168. https://doi.org/10.1016/j.biotechadv.2011.08.021.Secreted

U.S. Department of Health and Human Services. (2014). Healthy People 2020. Washington, D.C. Retrieved from http://www.healthypeople.gov/2020/about/default.aspx

Vadaparampil, S. T., Malo, T. L., Kahn, J. A., Salmon, D. A., Lee, J.-H., Quinn, G. P., ... Giuliano, A. R. (2014). Physicians' human papillomavirus vaccine recommendations, 2009 and 2011. American Journal of Preventive Medicine, 46(1), 80-4. https://doi.org/10.1016/j.amepre.2013.07.009

Wilson, R., Brown, D. R., Boothe, M. A. S., \& Harris, C. E. S. (2013). Knowledge and acceptability of the HPV vaccine among ethnically diverse Black women. Journal of Immigrant and Minority Health, 15(4), 747-57. https://doi.org/10.1007/s10903-012-9749-5

Winer, R. L., Lee, S.-K., Hughes, J. P., Adam, D. E., Kiviat, N. B., \& Koutsky, L. A. (2003). Genital Human Papillomavirus Infection: Incidence and Risk Factors in a Cohort of Female University Students. American Journal of Epidemiology, 157(3), 218-226. https://doi.org/10.1093/aje/kwf180

Author Information

* Dudith Pierre-Victor

National Cancer Institute, Division of Cancer Prevention

Bethesda, MD 20852

Phone: 240-276-7858

Email: dudith.pierre-victor@nih.gov

* corresponding author 


\section{Appendix I}

\section{Interview Guide Sample Questions}

Part A: HPV Knowledge

1. Have you ever heard of Human Papillomavirus or HPV?

2. Where/how did you hear about it? What is HPV?

Part B: Knowledge of HPV vaccine

1. Have you ever heard of Human Papillomavirus or HPV vaccine?

2. What do you know about the vaccine? Who should get it?

Part C: Attitudes

1. What do you think about the vaccine?

2. How do you feel about getting the vaccine?

Part D: Acceptability and Uptake

1. Have you initiated the HPV vaccine?

If yes: How many doses have you completed? At what age did you get vaccinated?

Part E: Factors influencing vaccine decision (If participants answered yes to Part D)

1. What/who motivated to get the vaccine? Walk me through the decision-making process.

2. Has a provider recommended that you take the vaccine? What did the provider say? What information did s/he provide? How did you (your parent) react following the recommendation?

Follow-up questions based on age at the time of recommendation and vaccination, individuals involved in decision-making, HPV-related conversations in the household, and sexuality-related conversations in the household.

Part F: Factors influencing vaccine decision (If participants answered no to Part D)

1. What/who do you think influenced/discouraged you to not get the vaccine? Walk me through the decision-making process.

2. Has a provider recommended that you take the vaccine? Do you have a primary healthcare provider? Have you ever asked your healthcare provider about HPV vaccine? Why (not)?

Follow-up questions based on age at the time of recommendation and vaccination, individuals involved in decision-making, HPV-related conversations in the household, and sexuality-related conversations in the household. 